\title{
Search Space Mapping: Getting a Picture of Coherent Laser Control
}

\author{
Janelle C. Shane, Vadim V. Lozovoy, and Marcos Dantus* \\ Department of Chemistry, Michigan State University, East Lansing, Michigan 48824
}

Received: June 26, 2006; In Final Form: August 17, 2006

\begin{abstract}
Search space mapping is a method for quickly visualizing the experimental parameters that can affect the outcome of a coherent control experiment. We demonstrate experimental search space mapping for the selective fragmentation and ionization of para-nitrotoluene and show how this method allows us to gather information about the dominant trends behind our achieved control.
\end{abstract}

Since the first demonstration by Assion et al., ${ }^{1}$ coherent laser control of chemical reactions using shaped femtosecond pulses has been the focus of many research efforts. In a typical control experiment, the electric field produced by a femtosecond pulse interacts with a molecule, producing ions and fragment ions that are detected using a mass spectrometer. The quantum control landscape for these problems is the set of all theoretically possible electric fields and their effects on the mass spectra. We will discuss the experimental case, in which the shape of the electric field can be controlled with a pulse shaper such as the design by Weiner, ${ }^{2}$ which can generate an enormous but finite number of possible combinations of phase and amplitude values over the frequencies within a pulse's spectrum. This set of all experimentally possible pulse shapes is the search space, and we seek to quickly gather experimental insights about the search space that will help guide exploration of these possibilities.

Learning algorithm search for pulses that produce control, first proposed by Judson and Rabitz, ${ }^{3}$ is a valuable strategy for managing the very large number of experimental parameters without prior knowledge of the physical processes involved. A fitness definition specifies what result is to be optimized, and the learning algorithm uses a form of feedback to search for pulse shapes with high fitness values. Although search is automated, a learning algorithm can be made more efficient by designing it with knowledge of the search space. Ideally, the goal is to discover a relationship to some known characteristic that allows pulse shapes to be directly designed, an approach used theoretically in optimal control theory. ${ }^{4}$

Here, we focus on knowledge that can be gained experimentally from the nonideal system being measured in the laboratory. For example, experimental determination of the prevalence and number of local and global maxima would help determine the degree of difficulty of the search and aid in the design of a learning algorithm that avoids suboptimal local maxima. Several general parameters, such as total intensity of the pulse's second harmonic generation (SHG) spectrum, could potentially play a role in determining experimental outcome. It would be helpful to quickly get a picture of the effects of these parameters, to

* E-mail: dantus@msu.edu. Tel: 517-355-9715 ×314. Fax: 517-3531793.

10.1021/jp0639997 CCC: \$33.50 determine which ones are important and which ones are not. In cases where exhaustive evaluation of a reduced set of pulse shapes provides a sufficient level of control, we want a way of mining this data set in its entirety to quickly determine the location of maxima and minima for different fragmentation pathways.

Search space mapping was discussed in the context of nonlinear optical control problems by Lozovoy and Dantus where they showed the first experimental search space map for the ionization and fragmentation of a large molecule. ${ }^{5}$ The same year, Baumert et al. mapped the search space for the ionization of potassium atoms, ${ }^{6}$ and Weinacht and co-workers studied the controlled fragmentation of $\mathrm{CH}_{2} \mathrm{BrI}$ and $\mathrm{CH}_{3} \mathrm{COCF}_{3}$, creating maps based on the two dimensions found after diagonalizing the experimentally evaluated control variable space. ${ }^{7}$ Here, we present how search space mapping, already shown to be a useful tool in problems involving shaping of nonlinear spectra ${ }^{8}$ can be used to gather insights into femtochemistry problems. We show how search space mapping provides a quick picture of the dominant effects and relationships in a control experiment, as well as a picture of search space noise level and roughness. We demonstrate search space maps for the problems of optimizing the intensities of different fragment ions produced by the interaction of intense phase-shaped pulses and paranitrotoluene and discuss what the maps reveal about the dominant effects governing its ionization and dissociation.

The first step in mapping the search space of a coherent control problem is to reduce this search space to a manageable number of dimensions and a manageable size. Most learning algorithms already restrict the number of control parameters (number of pixels, phases, and amplitude values) that are used. ${ }^{9,10}$ A second approach to search space reduction is parametrizing the phase functions in terms of a Taylor series expansion or a sinusoidal function. ${ }^{6,11}$ A third approach is to collect results from a parametrized search and reduce the space by principal control analysis. The resulting basis functions can be once again reoptimized.7,12 Our group has formulated a strategy based on nonlinear optical excitation and its dependence on the spectral phase of the excitation pulses. ${ }^{13,14}$ With an understanding of how to control multiphoton excitation (twoand three-photon), we realized that binary phase functions (no amplitude modulation, and only phase values of 0 or $\pi$ allowed) 
allow us to gain maximum control through multiphoton intrapulse interference with a minimum number of parameters. ${ }^{5,15}$ In addition to being robust and easy to reproduce, binary phase functions have shown to be a highly efficient approach toward finding significant control; with the 256 possible 8-bit binary phase functions, we can achieve 2 orders of magnitude of control over the relative yields of ortho-nitrotoluene fragments. ${ }^{16}$ The 256 different phase functions can be evaluated in under $5 \mathrm{~min}$, allowing us to repeat each experiment several times to check reproducibility and gain statistical information.

For this study, we used a Ti:sapphire oscillator and regenerative amplifier laser, capable of producing $35 \mathrm{fs}$ pulses of 0.8 $\mathrm{mJ}$ per pulse, at a $1 \mathrm{kHz}$ repetition rate. The beam was attenuated to $170 \mu \mathrm{J} /$ pulse and focused using a $50 \mathrm{~mm}$ lens into the vacuum chamber of a time-of-flight mass spectrometer. para-Nitrotoluene was introduced into the chamber to maintain a background pressure of $1 \times 10^{-5}$ Torr under fast flow conditions. Phase distortions common to all laser systems and sometimes introduced by optics were corrected at the sample using the multiphoton intrapulse interference phase scan (MIIPS) method, ${ }^{17,18}$ with the pulse shaper located before the amplifier. ${ }^{19}$ MIIPS allowed us to make sure our results are reproducible from one day to the next. Phase functions were applied using the same pulse shaper. The 8-bit functions were spread across the entirety of the spectrum, with the first and last bits covering the lowintensity wings of the spectrum.

In search space mapping, we see the entire search space at a glance by transforming the list of binary phase functions and their fitnesses into a two-dimensional colored map. To transform a binary phase function into an $(x, y)$ coordinate on a map, we first divide the phase function in two. The first half becomes the $x$-coordinate, and the second half becomes the $y$-coordinate. Defining the location of the most significant bit in each half of the sequence can affect the appearance of maps; ${ }^{8}$ here, we present the mapping method with the most straightforward visualization, in which the most significant bits of both the $x$ and $y$ halves of the sequence are located at the center of the phase function. Formally, the $x$ and $y$ coordinates of a particular 8-bit binary phase function $b_{1} \cdots b_{8}$ are given by $x=$ $\sum_{i=1}^{4} b_{i} 2^{i-1}$ and $y=\sum_{i=5}^{8} b_{i} 2^{8-i}$. The experimental fitness of each binary phase shape determines the color of the point at its corresponding $(x, y)$ coordinate $($ we chose red $=$ high fitness and black $=$ low fitness).

In Figure 1, we present a series of experimental search space maps in which the fitness criteria are (a) integrated SHG intensity, (b) the inverse of integrated SHG intensity, (d) integrated total ion intensity, (e) excitation favoring low versus high frequencies in the SHG spectrum, and (f) selective multiphoton excitation at the center frequency of the pulse's SHG spectrum while suppressing other frequencies. The integrated SHG (search space map shown in Figure 1a) is maximized for transform limited (TL) pulses, which appear in red at the extreme lower-left and upper-right corners of the map, and intermediate (yellow) for nearly flat phase functions, located along the edges of the maps. Heavily modulated phase functions have low-intensity second harmonic spectra; these pulse shapes appear in black and are found near the middle of the search space maps. Symmetric and antisymmetric phase functions are located along the diagonals of the maps. For the problem of producing a narrow peak in the center of the SHG spectrum (Figure 1f), symmetric or antisymmetric phase functions are optimal, so the diagonals appear red. In this mapping strategy, each row and column contains points corresponding to pulse

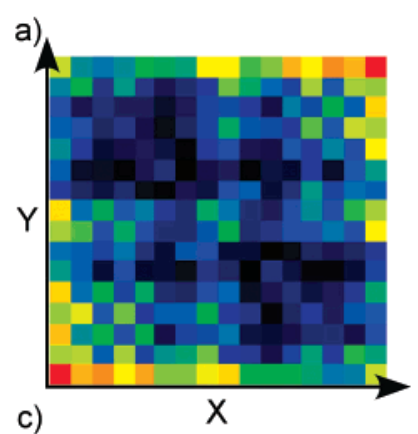

b)
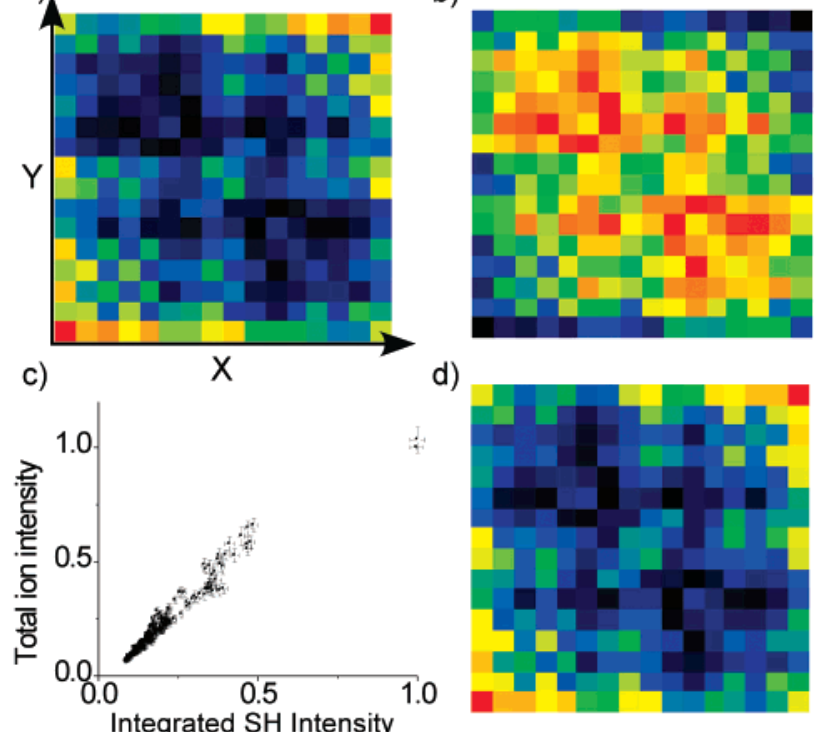

d)

e)
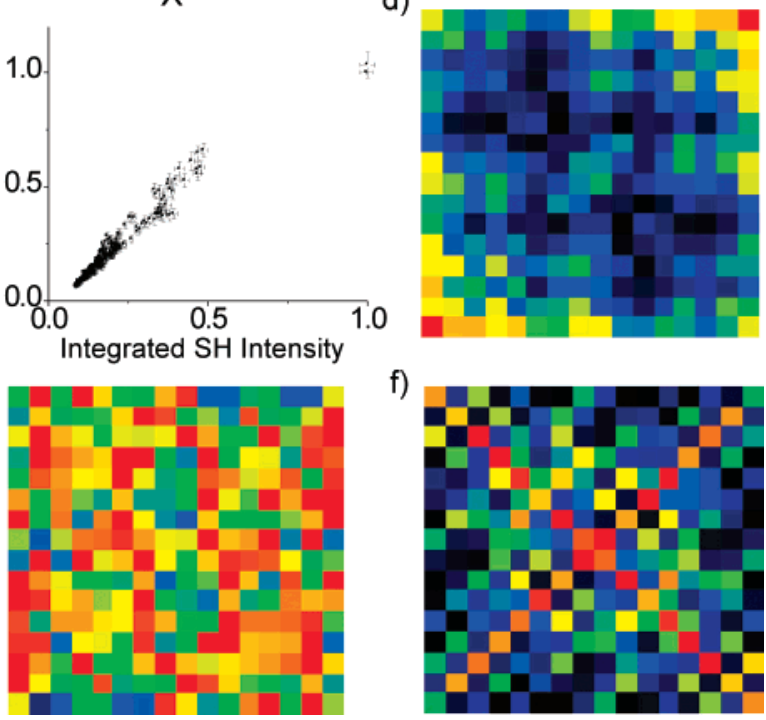

Figure 1. Search space maps in which the fitness criteria are as follows: (a) integrated SHG intensity, (b) the inverse of integrated SHG intensity, (d) integrated total ion intensity, (e) excitation favoring red versus blue frequencies, and (f) selective multiphoton excitation at the center frequency of the pulse while suppressing other frequencies. (c) Correlation plot of total $p$-nitrotoluene ion yield versus integrated SHG spectral intensity. For each map, red pixels correspond to pulse shapes that were most successful at optimizing a particular experimental outcome, while blue/black pixels were least successful. All maps show experimental results.

shapes that are at least $50 \%$ identical, with similar pulse shapes generally located close to each other.

The maps have rotational $\left(C_{2}\right)$ symmetry, due to the fact that a binary sequence and its dual (all 0 changed to $\pi$, and vice versa) should produce the same electric field except for the carrier phase. Deviation from rotational symmetry, visible at a glance, is a sign that there is either a systematic error (possibly in the shaper), or the noise is greater than the control. The effect of noise is seldom quantified in closed-loop experiments; however, it can play a very significant role. When we obtain symmetric search spaces, we are certain that the control observed is not dominated by noise and is therefore robust. In our case, we can reproduce our results for the 256 phases on different days and with different pulse shaping equipment.

Insights about coherent control search spaces can be gained at a glance by comparing their search space maps to those of known phenomena, revealing possible correlations and dominant effects. For example, panels a and d of Figure 1 are extremely similar, indicating a relationship between integrated ion signal and integrated SHG intensity. This linear relationship is confirmed by the correlation plot in Figure 1c.

Figure 2 shows the search space maps for the relative yields of $p$-nitrotoluene fragment ions, the average of 10 repetitions. The relative yield of an ion is the percent total ionization as defined by Watson. ${ }^{20}$ Some ions (Figure 2a) show inverse 


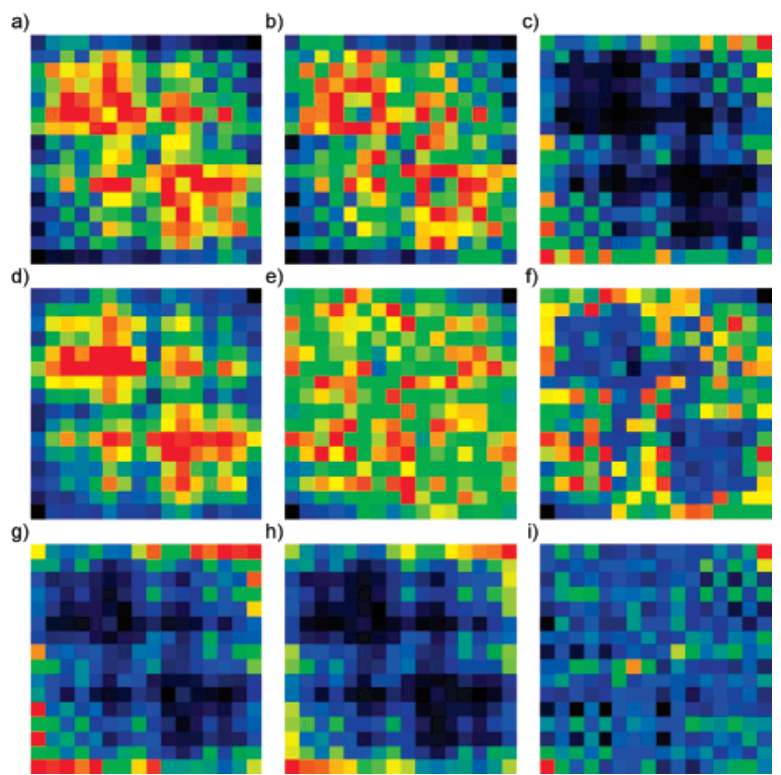

Figure 2. Experimentally obtained search space maps showing optimization of relative yields for various fragments of para-nitrotoluene, with $\mathrm{m} / z=$ (a) $12\left(\mathrm{C}^{+}\right)$; (b) $16\left(\mathrm{O}^{+}\right)$; (c) $28\left(\mathrm{CO}^{+}\right)$; (d) $30\left(\mathrm{NO}^{+}\right)$; (e) $39\left(\mathrm{C}_{3} \mathrm{H}_{3}{ }^{+}\right)$; (f) $51\left(\mathrm{C}_{4} \mathrm{H}_{3}{ }^{+}\right)$; (g) $65\left(\mathrm{C}_{5} \mathrm{H}_{5}{ }^{+}\right)$; (h) $91\left(\mathrm{C}_{7} \mathrm{H}_{7}{ }^{+}\right)$; (i) 107 $\left(\mathrm{C}_{7} \mathrm{H}_{7} \mathrm{O}^{+}\right)$.

relationships to total SHG intensity, as is evident when their search space maps are compared to an inverted map for total SHG intensity (Figure 1b). Others (Figure 2h) show direct relationships to total SHG intensity, while still others (Figure 2f) are intermediate cases, for which neither TL nor heavily modulated pulses are optimal. The maps show that, in general, heavily modulated pulses (producing low SHG intensity) maximize fragmentation, increasing the relative yield of small fragment ions. TL pulses minimize fragmentation, producing large fragment ions. The enhanced ionization and fragmentation model, based on a model proposed by Corkum and co-workers, ${ }^{21}$ may explain the tendency of TL pulses to minimize fragmentation. In this model, fragmentation requires that pulse duration is long enough for the molecule to undergo stretching, a requirement that is met by heavily modulated pulses but not by our $35 \mathrm{fs}$ TL pulses. An interesting exception to this trend is the fragment with $\mathrm{m} / \mathrm{z}=28$ (Figure 2c), which shows a direct relationship to total SHG intensity, a relationship that could be explained by the yield of a secondary reaction or the presence of $\mathrm{N}_{2}{ }^{+}$in addition to the fragment $\mathrm{CO}^{+}$. The correlation plots in Figure 3 show the relationship between total ion intensity (and therefore total SHG intensity), confirming the intuition gained from the maps.

Search space mapping sheds light on the laser-induced fragmentation mechanisms. Correlation diagrams as shown in Figure 3 (a, b, c, g, h) cannot be used to distinguish between multiphoton excitation and stimulated Raman transitions. The search space maps would have displayed circular structures if a stimulated Raman transition governed selective fragmentation. ${ }^{5}$ The corresponding search space maps are very similar to those obtained for total SHG and inverse SHG indicating a dependence on multiphoton excitation and not stimulated Raman transitions. If the dominant factor in selective fragmentation had been selective multiphoton excitation at a certain frequency, the search space maps would have shown diagonal lines as in Figure 1f, with the number and spacing of diagonal lines dependent on the frequency. ${ }^{5}$ If multiphoton selective excitation in the red or blue end of the spectrum had been important, the map would resemble Figure 1e. In this study of $p$-nitrotoluene, search space
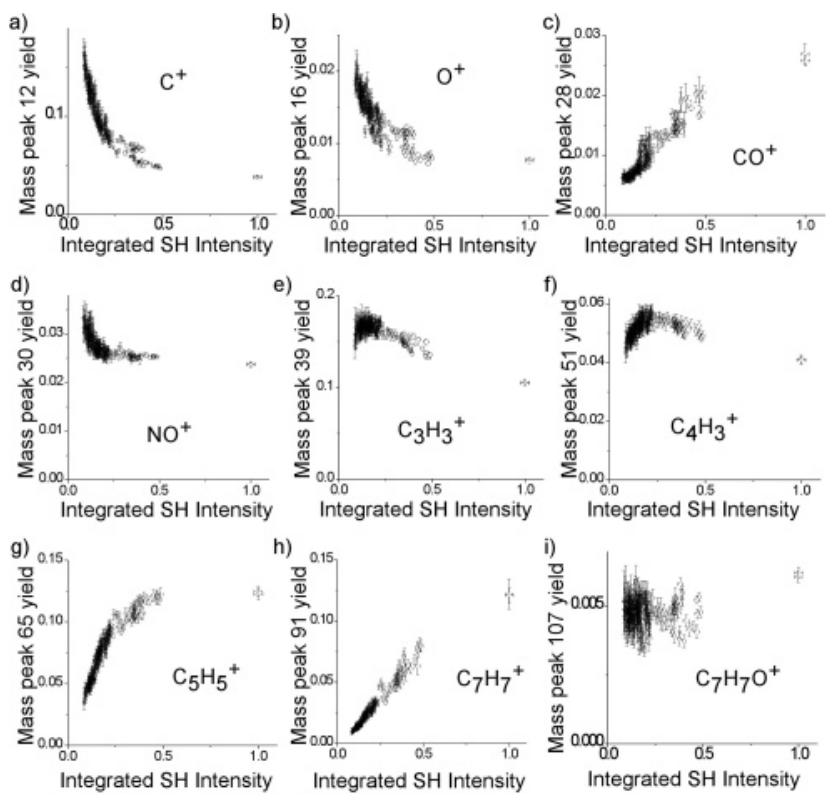

Figure 3. Correlation plots showing the experimentally obtained relationship between relative yields of various para-nitrotoluene fragments and total SHG spectral intensity. Error bars indicate the range of 1 standard deviation around the average of 10 trials.

mapping indicated that SHG intensity played a more important role in fragmentation than any of these other effects, helping us choose an intensity study as the most profitable next step.

Search space mapping also has advantages for dealing with experimental noise. Part of this advantage comes from the inherent dual symmetry in a search space map. The search space map for the selective production of $\mathrm{C}_{3} \mathrm{H}_{3}+$ (Figure 2e), for example, shows an inverse SHG intensity dependence despite the presence of noise, the latter evidenced by the lack of dual symmetry for most of the highest fitness values and confirmed by the error bars in the correlation plot (Figure 3e). Analyzing just the fittest phase shapes would have masked the dependence, since these highest-fitness phase shapes have varying SHG intensities. By examining a wide range of phase shapes at the same time, the general trend becomes apparent. Here, the search space map is more helpful than the correlation plot in highlighting the SHG intensity dependence, in part because the human eye tends to average out irregularities in a search space map, in the way that separate printed pixels create a blended image.

Search space mapping is a fast and flexible method for getting an overview of experimental results. This method can be applied to controlling relative yields of ions or to any problem for which a fitness function can be defined. Increasing the length of binary phase function used enhances detail, but very small maps also give useful information and take less time to experimentally evaluate, allowing the experiment to be repeated numerous times to provide statistical information. Through search space mapping, we can quickly see noise levels, as well as general trends behind the noise levels. A search space map shows which phase functions produce the highest fitnesses, as well as what they look like, as opposed to correlation plots. At a glance, search space mapping allows us to see relationships and effects that can guide future investigations or learning algorithm design.

Acknowledgment. Support for this research comes from the National Science Foundation CHE- 0500661, and is gratefully acknowledged. We appreciate the work of Michael Kangas who acquired the data for this work. We also appreciate the help 
from Dr. Tissa Gunaratne who compiled the data and helped in the preparation of the manuscript.

\section{References and Notes}

(1) Assion, A.; Baumert, T.; Bergt, M.; Brixner, T.; Kiefer, B.; Seyfried, V.; Strehle, M.; Gerber, G. Science 1998, 282, 919.

(2) Weiner, A. M. Rev. Sci. Instrum. 2000, 71, 1929.

(3) Judson, R. S.; Rabitz, H. Phys. Rev. Lett. 1992, 68, 1500.

(4) Rice, S. A.; Zhao, M. In Optical Control of Molecular Dynamics; Wiley: New York, 2000; p 437.

(5) Lozovoy, V. V.; Dantus, M. ChemPhysChem 2005, 6, 1970.

(6) Wollenhaupt, M.; Prakelt, A.; Sarpe-Tudoran, C.; Liese, D.; Baumert, T. J. Mod. Opt. 2005, 52, 2187.

(7) Cardoza, D.; Trallero-Herrero, C.; Langhojer, F.; Rabitz, H.; Weinacht, T. J. Chem. Phys. 2005, 122, 124306.

(8) Lozovoy, V. V.; Shane, J. C.; Xu, B. W.; Dantus, M. Opt. Express 2005, 13, 10882.

(9) Graham, P.; Menkir, G.; Levis, R. J. Spectrochim. Acta, Part B 2003, 58, 1097.

(10) Brixner, T.; Kiefer, B.; Gerber, G. Chem. Phys. 2001, 267, 241.
(11) Herek, J. L.; Wohlleben, W.; Cogdell, R. J.; Zeidler, D.; Motzkus, M. Nature (London) 2002, 417, 533.

(12) Wells, E.; Betsch, K. J.; Conover, C. W. S.; DeWitt, M. J.; Pinkham, D.; Jones, R. R. Phys. Rev. A 2005, 72, art. no. 063406.

(13) Walowicz, K. A.; Pastirk, I.; Lozovoy, V. V.; Dantus, M. J. Phys. Chem. A 2002, 106, 9369.

(14) Lozovoy, V. V.; Pastirk, I.; Walowicz, K. A.; Dantus, M. J. Chem Phys. 2003, 118, 3187.

(15) Comstock, M.; Lozovoy, V. V.; Pastirk, I.; Dantus, M. Opt. Express 2004, 12, 1061 .

(16) Lozovoy, V. V.; Gunaratne, T. C.; Shane, J. C.; Dantus, M. ChemPhysChem, in press.

(17) Xu, B. W.; Gunn, J. M.; Dela Cruz, J. M.; Lozovoy, V. V.; Dantus, M. J. Opt. Soc. Am. B 2006, 23, 750.

(18) Lozovoy, V. V.; Pastirk, I.; Dantus, M. Opt. Lett. 2004, 29, 775.

(19) Lozovoy, V. V.; Dantus, M. Annu. Rep. Prog. Chem. C 2006, 102, 227.

(20) Watson, J. T. Introduction to Mass Spectrometry, 3rd ed.; Lippincott-Raven Publishers: Philadelphia, PA, 1997.

(21) Ivanov, M.; Seideman, T.; Corkum, P.; Ilkov, F.; Dietrich, P. Phys. Rev. A 1996, 54, 1541 . 